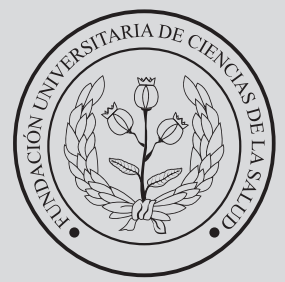

FUCS

\section{Re per
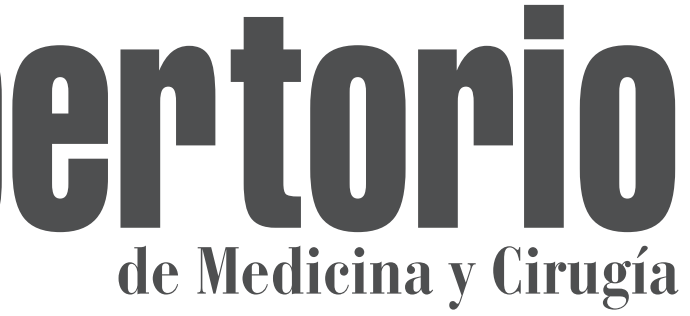

20

(Núm. Supl.1)

2020

Artículo de investigación

\title{
Conglomerados que favorecen la transmisión del COVID-19 en Colombia
}

Jorge Enrique Díaz-Pinzón ${ }^{a}$

\section{Clusters that facilitate COVID-19 transmission in Colombia}

a Ingeniero. Magister en Gestión de la Tecnologia Educativa, Especialista en Administración de la Informática Educativa. Docente de matemáticas e Investigador, Secretaría de Educación de Soacha, Cundinamarca.

\section{R E S U M EN}

Introducción: a fines de 2019 se informó la infección con un nuevo beta coronavirus, posteriormente llamado SARS-CoV-2, en personas que habían estado expuestas a un mercado de mariscos en Wuhan, China, donde se vendían animales vivos. Desde entonces ha habido una rápida propagación del virus, lo que ha provocado una pandemia mundial de COVID-19. Objetivo: analizar estadísticamente los conglomerados en Colombia generados por el COVID-19. Metodología: esta investigación se centró en la población de conglomerados y dentro de estos su número, casos confirmados, muestras tomadas, fallecidos y porcentaje de positividad para COVID-19, con la información proveniente del Instituto Nacional de Salud. Resultados: el conglomerado presenta el mayor número con un total de 481, seguido de instituciones de salud con 320. Se aprecia que el conglomerado FFMM presenta el mayor número de casos confirmados con un total de 12.555, seguido de empresa con 5.887. El conglomerado FFMM presenta el mayor número de muestras tomadas un total de 29.781, seguido de empresa con 27.118. Asimismo, el conglomerado centro de protección presenta el mayor número de fallecidos con un total de 185 , seguido de FFMM con 64; el conglomerado FFMM presenta el mayor número de porcentaje de positividad con un total de 42,16, seguido de población carcelaria con 39,29. Conclusión: los conglomerados deben aproximarse a algunas condiciones, entre las que están la cercanía, las personas, el tiempo de permanencia y la inestabilidad de las medidas de protección, además de la concentración de personas en espacios cerrados.

Palabras clave: COVID-19, conglomerados, SARS-CoV-2, pandemia.

(C) 2020 Fundación Universitaria de Ciencias de la Salud - FUCS. Este es un artículo Open Access bajo la licencia CC BY-NC-ND (http://creativecommons.org/licenses/by-nc-nd/4.0/)

\section{INFORMACIÓN DEL ARTÍCULO}

Historia del artículo:

Fecha recibido: noviembre 3 de 2020 Fecha aceptado: noviembre 12 de 2020
Autor para correspondencia. Ing. Jorge Enrique Díaz Pinzón jediazp@unal.edu.co
DOI

10.31260/RepertMedCir.01217372.1131 
Introduction: in late 2019, a novel beta coronavirus, later known as SARS-CoV-2, infection was reported in people who had been exposed to a seafood market in Wuhan, China, where live animals were sold. Since then there has been a rapid spread of the virus, causing a global COVID-19 pandemic. Objective: to conduct a statistical analysis of clusters of COVID-19 transmission in Colombia. Methodology: this research was focused on COVID-19 clusters, their size, confirmed cases, testing, deaths and positivity rate, from information obtained by the National Institute of Health. Results: the companies setting accounts for the greatest number of clusters of COVID-19 transmission, 481, followed by 320 in healthcare institutions. It was observed that the armed forces cluster had the highest number of confirmed cases reaching a total of 12.555, followed by 5.887 in companies. COVID-19 testing was conducted mostly in the armed forces in 29.781 individuals, followed by the companies in 27.118. Likewise, the highest number of fatalities occurred in the welfare center cluster reaching a total of 185 , followed by 64 in the armed forces; the armed forces cluster had the highest COVID-19 positivity rate of $42.16 \%$, followed by $39.29 \%$ in the prison system. Conclusion: clusters must address some conditions, such as proximity, people, length of stay, and instability of COVID-19 protective measures, in addition to the density of people in confined spaces.

Key words: COVID-19, conglomerados, SARS-CoV-2, pandemia.

(C) 2020 Fundación Universitaria de Ciencias de la Salud - FUCS. This is an open access article under the CC BY-NC-ND license (http://creativecommons.org/licenses/by-nc-nd/4.0/).

\section{INTRODUCCIÓN}

A finales de 2019, se informó de la infección con un nuevo beta coronavirus en personas que habían estado expuestas a un mercado de mariscos en Wuhan, China, donde se vendían animales vivos. Desde entonces, ha habido una rápida propagación del virus, lo que ha provocado una pandemia mundial de COVID-19. ${ }^{1}$

El SARS-CoV-2 está relacionado con los coronavirus de murciélago y con el SARS-CoV-1, el virus que causa el síndrome respiratorio agudo severo (SARS). ${ }^{2} \mathrm{Al}$ igual que el SARS-CoV-1, el SARS-CoV-2 ingresa a las células humanas a través del receptor de la enzima convertidora de angiotensina 2 (ACE2). ${ }^{3}$

El SARS-CoV-2 se transmite principalmente de persona a persona a través de gotitas respiratorias, que generalmente se liberan cuando una persona infectada tose o estornuda. Debido a que las gotas generalmente caen a unos pocos metros, la probabilidad de transmisión disminuye si las personas permanecen separadas al menos dos metros. ${ }^{4}$

Se ha detectado ARN del SARS-CoV-2 en sangre y heces, aunque no se ha documentado la diseminación fecal-oral. El SARS-CoV-2 puede persistir en cartón, plástico y acero inoxidable durante días. ${ }^{5,6}$ Como resultado, la contaminación de superficies inanimadas puede jugar un papel en la transmisión. ${ }^{4,7}$

Un desafío importante para contener la propagación del SARS-CoV-2 es que las personas presintomáticas son infecciosas. ${ }^{8}$ Informes recientes sugieren que los pacientes pueden ser infecciosos de 1 a 3 días antes del inicio de los síntomas y que hasta un 40 a $50 \%$ de los casos pueden atribuirse a la transmisión de personas asintomáticas o presintomáticas. ${ }^{4,9}$ Justo antes o poco después de la aparición de los síntomas, los pacientes tienen niveles altos de virus nasofaríngeos, que luego disminuyen en el transcurso de aproximadamente 1 semana..$^{10}$ Los pacientes con enfermedad grave pueden excretar el virus durante períodos más prolongados, aunque no está clara la duración de la excreción viral infecciosa. ${ }^{11}$

El período medio de incubación desde la exposición hasta el inicio de los síntomas es cerca de 4 a 5 días y $97,5 \%$ de los pacientes con síntomas los presentarán dentro de 11,5 días posteriores a la infección. ${ }^{12}$ Pueden incluir fiebre, tos, dolor de garganta, malestar y mialgias. Algunos pacientes presentan síntomas gastrointestinales, como anorexia, náuseas y diarrea. ${ }^{13}$

\section{METODOLOGÍA}

El trabajo de investigación se efectuó mediante un enfoque cuantitativo: la investigación cuantitativa es aquella en la que se almacenan y exploran datos sobre variables, y estudia las propiedades y fenómenos cuantitativos, según Hurtado y Toro (1998) citado por Díaz. ${ }^{14}$

\section{Población}

Esta investigación se centró en la población de conglomerados y dentro de estos su número casos confirmados, muestras tomadas, fallecidos y porcentaje de positividad para COVID-19, con la información proveniente del Instituto Nacional de Salud ${ }^{15}$, en el periodo comprendido entre el 6 de marzo al 1 de noviembre de 2020 (tabla 1). 
Tabla 1. Conglomerados por COVID-19

\begin{tabular}{|l|c|c|c|c|c|}
\hline \multicolumn{1}{|c|}{ Conglomerados } & Número de conglomerados & Casos confirmados & Muestras tomadas & Fallecidos & \% positividad \\
\hline Empresa & 481 & 5887 & 27118 & 28 & 21,71 \\
\hline Instituciones de salud & 320 & 3780 & 17100 & 42 & 22,11 \\
\hline Policía & 167 & 2012 & 5426 & 6 & 37,08 \\
\hline Centros de protección & 151 & 3667 & 9667 & 185 & 37,93 \\
\hline FFMM & 111 & 12555 & 29781 & 64 & 42,16 \\
\hline Población carcelaria & 110 & 4698 & 11958 & 15 & 39,29 \\
\hline Indígenas & 40 & 432 & 1233 & 9 & 35,04 \\
\hline Comunitario & 16 & 511 & 1938 & 22 & 26,37 \\
\hline Plazas de mercado & 15 & 786 & 2011 & 32 & 39,09 \\
\hline
\end{tabular}

Fuente: el autor

\section{RESULTADOS}

En la figura 1 se observa que el conglomerado empresa presenta el mayor número con un total de 481, seguido de instituciones de salud con 320 , policía 167 , centros de protección 151, FFMM 111, población carcelaria 110, indigenas 40, comunitario 16, y plazas de mercado 15.
En la figura 2 se aprecia que el conglomerado FFMM presenta el mayor número de casos confirmados con un total de 12.555, seguido de empresa 5.887, población carcelaria 4.698, instituciones de salud 3.780, centros de protección 3.667, policía 2.012, plazas de mercado 786, comunitario 511 e indigenas 432 .

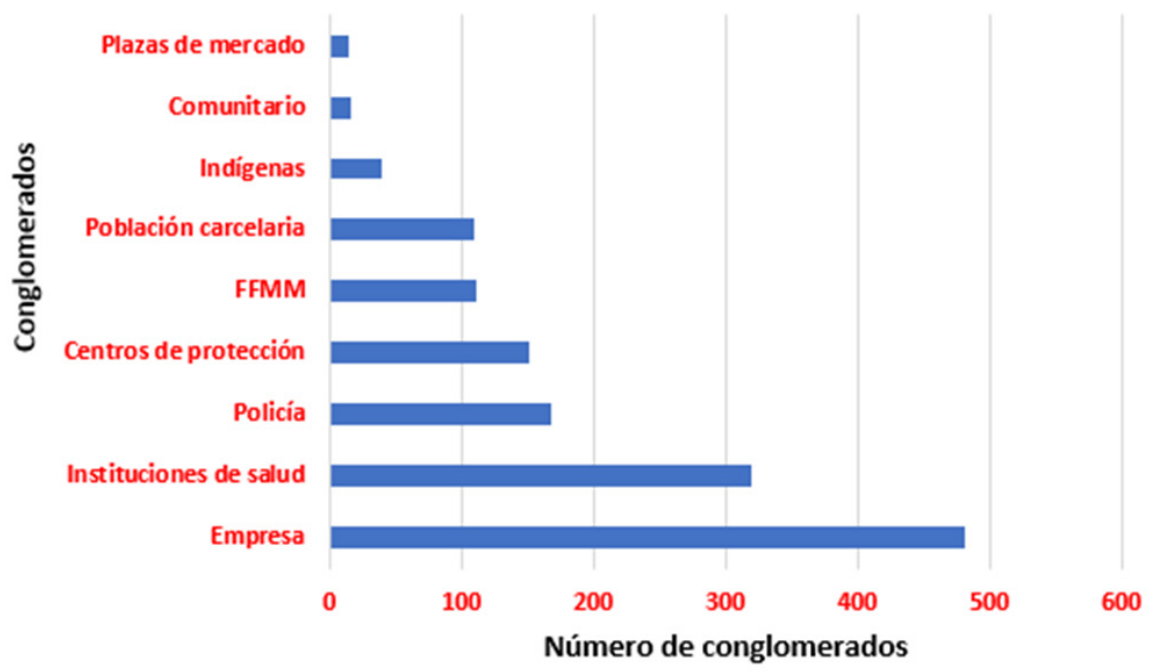

Figura 1. Número de conglomerados. Fuente: el autor.

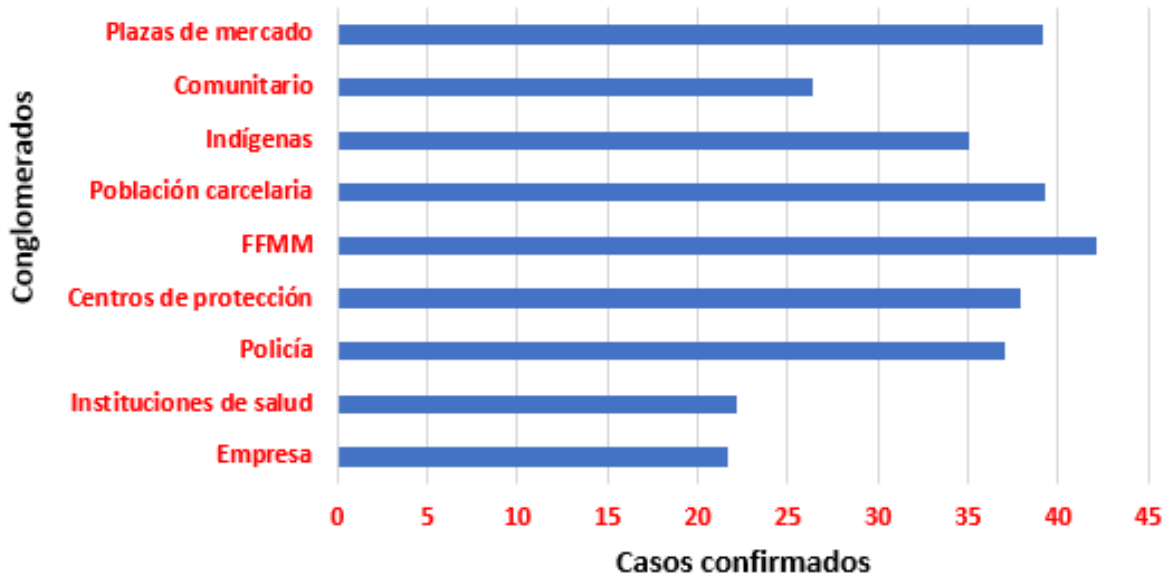

Figura 2. Casos confirmados por conglomerados. Fuente: el autor. 
En la figura 3 se describe que el conglomerado FFMM presenta el mayor número de muestras tomadas con un total de 29.781, seguido de empresa 27.118, instituciones de salud 17.100, población carcelaria 11.958 , centro de protección 9.667, policía 5.426, comunitario 1.938 , plazas de mercado 2.011 e indigenas 1.233 .
En la figura 4 se observa que el conglomerado centro de protección presenta el mayor número de fallecidos con un total de 185, seguido de FFMM 64, instituciones de salud 42, plazas de mercado 32, empresa 28, comunitario 22, población carcelaria 15, indígenas 9 y policía 6 .

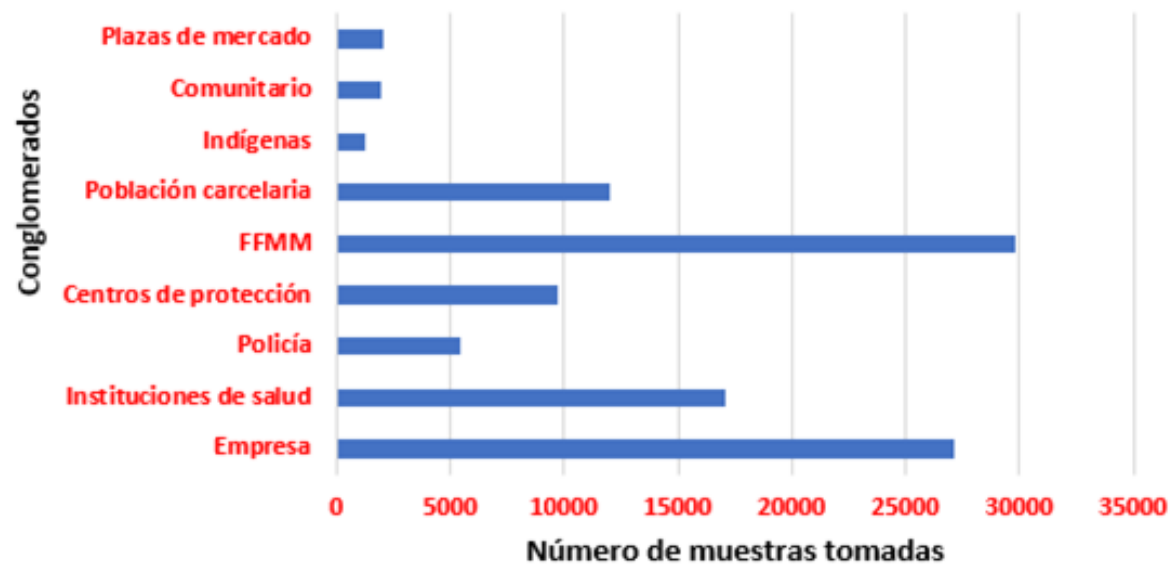

Figura 3. Número de muestras tomadas por conglomerados. Fuente: el autor.

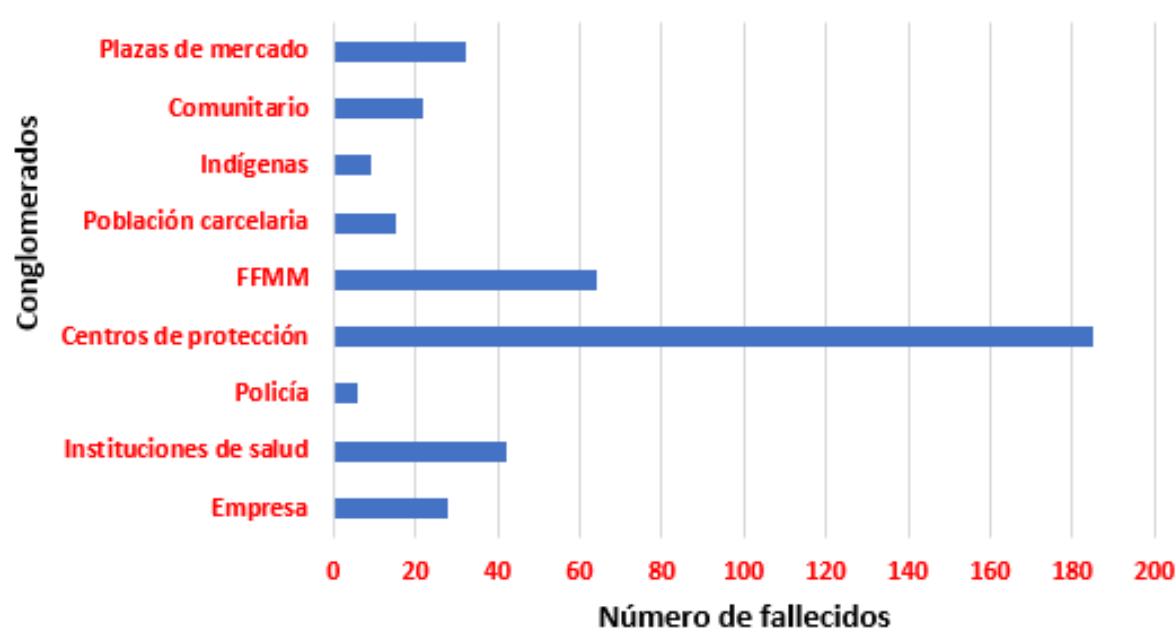

Figura 4. Número de fallecidos por conglomerados. Fuente: el autor.

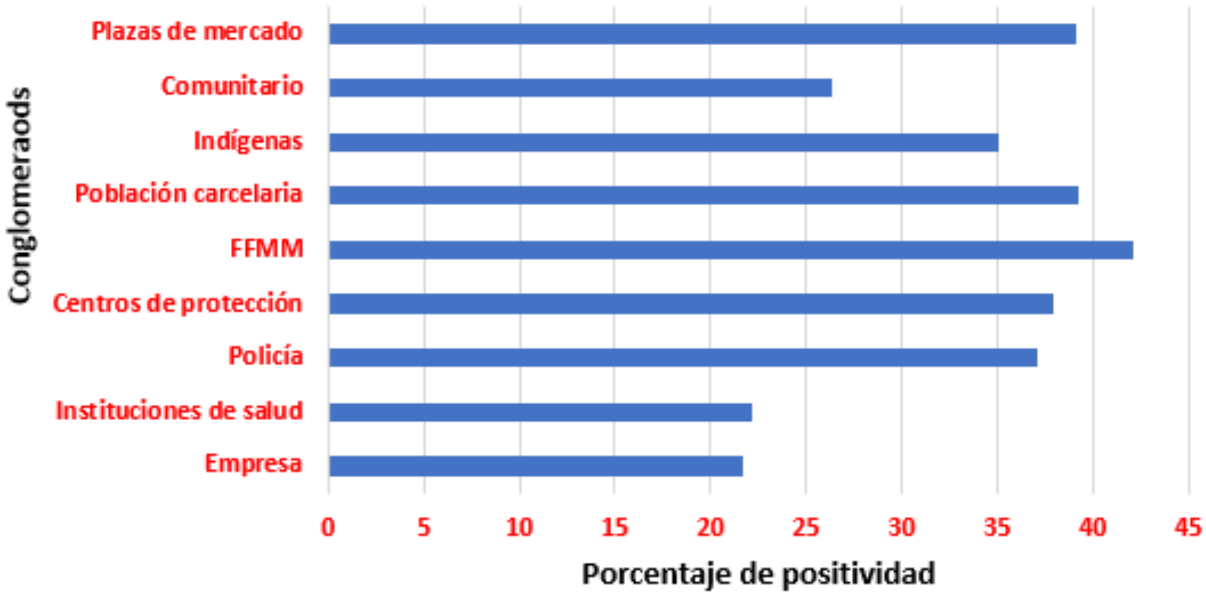

Figura 5. Porcentaje de positividad por conglomerados. Fuente: el autor. 
En la figura 5 se aprecia que el conglomerado FFMM presenta el mayor porcentaje de positividad con un total de 42,16, seguida de población carcelaria 39,29, plazas de mercado 39,09, centro de protección 37,93, policía 37,08, indigenas 35,04, comunitario 26,37, instituciones de salud 22,11 y empresa 21,71 .

\section{CONCLUSIONES}

De acuerdo con la información expuesta anteriormente en los cuales por sus características y factores que benefician su transmisión, el nuevo coronavirus circula con mayor facilidad. Podemos determinar que el conglomerado empresa presenta el mayor número con un total de 481, seguido de instituciones de salud con 320, policía 167. Además, se aprecia que el conglomerado FFMM presenta el mayor número de casos confirmados con un total de 12.555 , empresa con 5.887, seguido de población carcelaria 4.698. De igual forma, el conglomerado FFMM presenta el mayor número de muestras tomadas con un total de 29.781, seguido de empresa con 27.118, instituciones de salud 17.100. El conglomerado centro de protección presenta el mayor número de fallecidos con un total de 185, seguido de FFMM con 64, instituciones de salud 42. El conglomerado FFMM presenta el mayor número de porcentaje de positividad con un total de 42,16 , seguido de población carcelaria con 39,29 y plazas de mercado 39,09.

Los conglomerados deben aproximarse a algunas condiciones, entre las que están la cercanía, las personas, el tiempo de permanencia y la inestabilidad de las medidas de protección, además de la concentración de personas en espacios cerrados.

Acorde con Chaccour citado por Díaz ${ }^{16}$, no obstante las múltiples incertidumbres que a nivel biológico, clínico y epidemiológico subsisten en relación con este nuevo virus, lo que ya es claro es que cada país ha reconocido o está reconociendo a la misma amenaza con diferentes medidas y/o con una mitigación diferente.

\section{CONFLICTO DE INTERÉS}

El autor declara no tener ningún conflicto de interés.

\section{REFERENCIAS}

1. Gandhi RT, Lynch JB, Del Rio C. Mild or Moderate Covid-19. N Engl J Med. 2020;383(18):1757-66. doi: 10.1056/NEJMcp2009249

2. Zhu N, Zhang D, Wang W, Li X, Yang B, Song J, et al. A Novel Coronavirus from Patients with Pneumonia in China, 2019. N Engl J Med. 2020;382(8):727-33. doi: 10.1056/NEJMoa2001017
3. Hoffmann M, Kleine-Weber H, Schroeder S, Kruger N, Herrler T, Erichsen S, et al. SARS-CoV-2 Cell Entry Depends on ACE2 and TMPRSS2 and Is Blocked by a Clinically Proven Protease Inhibitor. Cell. 2020;181(2):271-80 e8. doi:10.1016/j.cell.2020.02.052

4. Wei WE, Li Z, Chiew CJ, Yong SE, Toh MP, Lee VJ. Presymptomatic Transmission of SARS-CoV-2 - Singapore, January 23-March 16, 2020. MMWR Morbidity and mortality weekly report. 2020;69(14):411-5. doi: 10.15585 / mmwr.mm6914el

5. van Doremalen N, Bushmaker T, Morris DH, Holbrook MG, Gamble A, Williamson BN, et al. Aerosol and Surface Stability of SARS-CoV-2 as Compared with SARS-CoV-1. N Engl J Med. 2020;382(16):1564-7. D doi: 10.1056/NEJMc2004973.

6. Kampf G, Todt D, Pfaender S, Steinmann E. Persistence of coronaviruses on inanimate surfaces and their inactivation with biocidal agents. The Journal of hospital infection. 2020;104(3):24651. Epub 2020/02/10.

7. Centers for Disease Control and Prevention. Coronavirus Disease 2019 (COVID-19) [Internet]. Washington, D.C. : Department of Health and Human Services; 2020 [cited 2020 octubre]; Available from: https://www.cdc.gov/other/language-assistance. html\#Spanish.

8. Kimball A, Hatfield KM, Arons M, James A, Taylor J, Spicer K, et al. Asymptomatic and Presymptomatic SARS-CoV-2 Infections in Residents of a Long-Term Care Skilled Nursing Facility King County, Washington, March 2020. MMWR Morbidity and mortality weekly report. 2020;69(13):377-81. doi: 10.15585/ mmwr.mm6913el.

9. He X, Lau EHY, Wu P, Deng X, Wang J, Hao X, et al. Temporal dynamics in viral shedding and transmissibility of COVID-19. Nature medicine. 2020;26(5):672-5. doi: 10.1038/s41591-020-0869-5.

10. Wolfel R, Corman VM, Guggemos W, Seilmaier M, Zange S, Muller MA, et al. Virological assessment of hospitalized patients with COVID-2019. Nature. 2020;581(7809):465-9. doi: 10.1038/s41586020-2196-x.

11. Liu Y, Yan LM, Wan L, Xiang TX, Le A, Liu JM, et al. Viral dynamics in mild and severe cases of COVID-19. The Lancet Infectious diseases. 2020;20(6):656-7. Epub 2020/03/23.

12. Lauer SA, Grantz KH, Bi Q, Jones FK, Zheng Q, Meredith HR, et al. The Incubation Period of Coronavirus Disease 2019 (COVID-19) From Publicly Reported Confirmed Cases: Estimation and Application. Annals of internal medicine. 2020;172(9):577-82.

13. Wang $\mathrm{D}, \mathrm{Hu} \mathrm{B}, \mathrm{Hu} \mathrm{C}$, Zhu F, Liu X, Zhang J, et al. Clinical Characteristics of 138 Hospitalized Patients With 2019 Novel Coronavirus-Infected Pneumonia in Wuhan, China. Jama. 2020;323(11):1061-9. Epub 2020/02/08.

14. Díaz-Pinzón JE. Simulador Applet Descartes: Como didáctica de enseñanza de la función cuadrática. INNOVA Research Journal 2017;2(8):69-78.

15. Instituto Nacional de Salud. Coronavirus (COVID-2019) en Colombia[Internet] Colombia: Instituto Nacional de Salud; 2020 [cited 2020 noviembre 2]; Available from: https://www.minsalud. gov.co/salud/publica/PET/Paginas/Covid-19_copia.aspx.

16. Díaz-Pinzón JE. Precisión del pronóstico de la propagación del COVID-19 en Colombia. Repert Med Cir. 2020;29(Núm. Supl.1):2733. doi: 10.31260/RepertMedCir.01217372.1045. 